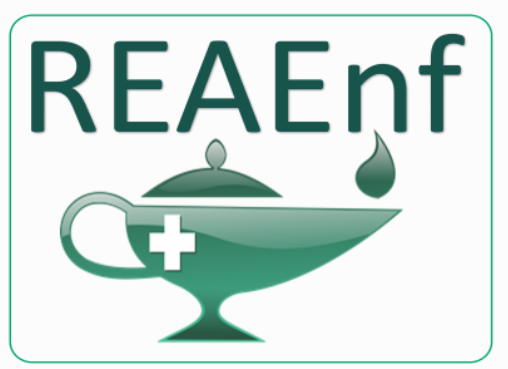

Revista Eletrônica Acervo Enfermagem

\section{REVISÃO BIBLIOGRÁFICA}

Recebido em: 11/2019

Aceito em: 12/2019

Publicado em: 2/2020

\title{
Depressão em professores: revisão integrativa da literatura
}

\author{
Depression among teachers: integrative literature review
}

\author{
Depresión en los profesores: revisión integrativa de la literatura
}

Paloma Calazans Galinari ${ }^{*}$, Jonathan Mendes de Castro ${ }^{1}$, Ranyelli Estefany da Costa Martins ${ }^{1}$, Nara Pereira de Faria Carvalho de Alencar ${ }^{1}$, Marcela Alves Azevedo ${ }^{1}$, Talles Vinícius de Castro Oliveira $^{1}$, Enaile de Souza Proti ${ }^{1}$, Diego Azevedo Araújo ${ }^{1}$, Carlos Henrique Wernersbach Guerra ${ }^{1}$, Wendel Jose Teixeira Costa ${ }^{1,2}$.

\begin{abstract}
Resumo: Essa pesquisa buscou reunir e sistematizar resultados de investigações científicas relacionadas à prevalência de depressão em professores. Trata-se de uma revisão integrativa da literatura sobre a prevalência de depressão em professores. A coleta de dados ocorreu em setembro de 2019 e foram utilizadas as bases de dados eletrônicas Literatura Latino-Americana em Ciências da Saúde (LILACS) e US National Library of Medicine (PubMed), com os descritores depressão, professores e saúde ocupacional, no idioma inglês, combinados por meio do operador booleano (AND). A amostra final foi constituída por cinco artigos, sendo dois artigos da PubMed e três da LILACS, as temáticas centrais foram prevalência da depressão entre professores e afastamento do trabalho relacionado à depressão. A literatura analisada demonstra maior prevalência de depressão e sintomas depressivos em professores nas idades acima de 40 anos, no sexo feminino. Também se destaca como a principal causa de afastamento do trabalho relacionado a transtornos mentais.
\end{abstract}

Palavras-chave: Depressão, Professores, Saúde ocupacional.

\begin{abstract}
This research aimed to gather and systematize results of scientific research related to the prevalence of depression in teachers. This is an integrative literature review about the prevalence of depression in teachers. Data collection took place in September 2019 and the electronic databases Literatura Latino-Americana em Ciências da Saúde (LILACS) e US National Library of Medicine (PubMed) were used, with the descriptors depression, teachers and occupational health, in English, combined through the Boolean operator (AND). The final sample consisted of five articles, two articles from PubMed and three from LILACS. The central themes were prevalence of depression among teachers and absence from work related to depression. The literature reviewed shows a higher prevalence of depression and depressive symptoms in female teachers over 40 years of age. It also stands out as the main cause of sick leave related to mental disorders.
\end{abstract}

Keywords: Depression, Teachers, Occupational health.

Resumen: Esta investigación tuvo como objetivo reunir y sistematizar los resultados de la investigación científica relacionada con la prevalencia de la depresión en los docentes. Esta es una revisión integradora de la literatura la prevalencia de la depresión en los docentes. La recopilación de datos tuvo lugar en septiembre de 2019 y se utilizaron las bases de datos electrónicas Literatura Latino-Americana em Ciencias da Salude (LILACS) y US National Library of Medicine (PubMed), con los descriptores de depresión,

${ }^{1}$ Centro Universitário de Caratinga (UNEC), Caratinga - MG. *E-mail: palomagalinarii@hotmail.com

${ }^{2}$ Lab. de Escrita Científica, Escola Superior de Ciências da Santa Casa de Misericórdia (EMESCAM), Vitória - ES. 
docente y salud laboral, en inglés, combinados a través del operador booleano (AND). La muestra final consistió en cinco artículos, dos artículos de PubMed y tres de LILACS. Los temas centrales fueron la prevalencia de depresión entre los docentes y la ausencia del trabajo relacionada con la depresión. La literatura revisada muestra una mayor prevalencia de depresión y síntomas depresivos en maestras mayores de 40 años. También se destaca como la principal causa de baja por enfermedad relacionada con trastornos mentales.

Palabras clave: Depresión; Maestros; Salud Laboral.

\section{INTRODUÇÃO}

A depressão é considerada um transtorno mental, com um conjunto de sintomas fisiológicos e psicológicos, com intensidade que varia de severa a leve, apresentando de forma episódica ou continua. É acompanhada pela incapacidade de fazer tarefas diárias, por no mínimo duas semanas, também pode ser definida por tristeza persistente, humor deprimido e a perda de interesse em afazeres que geralmente são satisfatórias. É classificado pela psicopatologia como transtorno de humor e afetivo, sendo caracterizada como uma emoção dominante que traz percepções do indivíduo em relação ao mundo e que as experiencias originadas da relação com o meio, fazem com o que todos momentos vividos sejam visualizados como evitável e negativo (ASSOCIAÇÃO AMERICANA DE PSIQUIATRIA, 2014).

Quanto aos sintomas de depressão, podem ser separados em sintomas físicos, sintomas emocionais, sintomas motivacionais e sintomas cognitivos. Os sintomas físicos se manifestam por redução da atividade física, aumento ou diminuição do apetite e sono e fadiga. Os sintomas emocionais se manifestam pelo sentimento de tristeza, abatimento, desesperança, infelicidade, choro constante, perde o interesse pelos familiares, lazer, do prazer com a vida, podendo em casos graves se isolar e desenvolver pensamentos suicidas. Os sintomas motivacionais ocorrem quando a pessoa apresenta baixa energia e redução da iniciativa de executar funções básica para sobrevivência. Quanto aos sintomas cognitivos apresentam sensação de incapacidade, culpa, perda de memória, ideias pessimistas, diminuição na capacidade de solucionar conflitos (ATKINSON LR, et al. 2002).

Daqui a 20 anos, a depressão será umas das maiores causas de enfermidades no mundo perdendo somente para doenças cardiovasculares, de acordo com estudos epidemiológicos. (NASCIMENTO CDC, et al., 2013). No ambiente de trabalho a depressão é uma das doenças mais frequentes. Devido a estarem a frente em várias situações e problemas complicados, os trabalhadores ficam com energias tanto mentais a físicas reduzidas, o que traz sensações negativas em relação o desenvolvimento no emprego. Boa parte dos trabalhadores sofrerá depressão de forma grave que terão a necessidade de serem afastados (LASSER J e KAHN J, 1998; NOGUEIRA DXP, 2012). A depressão foi a principal causa de incapacidade no ano de 2000 segundo a Organização Mundial de Saúde (OMS), e demonstrou ser grande contribuinte para o desenvolvimento de outras doenças. Segundo a mesma a depressão tende a alcançar o segundo lugar no ranking do Disability Adjusted Life Years (DALYs) até o ano de 2020, ressaltando a depressão como um problema de saúde pública a nível mundial (ORGANIZAÇÃO MUNDIAL DE SAÚDE, 2019).

Os transtornos mentais de acordo com levantamentos realizados pela Previdência Social desde 2008 respondem pela terceira causa de afastamento do trabalho no Brasil. No trabalho, a sobrecarga e o conflito de interesses, contribuem para o esgotamento e o desiquilíbrio, que levam ao agravamento da saúde mental manifestada principalmente pela depressão, provocando disfunção laboral e sofrimento intenso (BOURBONNAIS R, et al., 1998). Algumas das pressões que os professores sofrem como pressões profissionais, econômicas e institucionais interferem na duração da carreira. (JOHNSRUD LK e HECK RH, 1998). Um dos principais fatores de distúrbios de saúde entre os professores é devido ao fato de serem mal remunerados, essa condição faz com que os professores sejam obrigados a trabalhar muitas vezes em mais de uma instituição de ensino ou tenham que fazerem longas jornadas de trabalho (GATTI BA, et al., 2011).

Professores depressivos no ambiente escolar, fazem com que o ensino fique pesado e negativo, trazendo consequências subestimadas, os sintomas de depressão fazem com oque tenham dificuldade de 
se envolver de forma positiva com seus alunos e de transmitir um ensino com qualidade. Educadores com emocional desregulado tem mais dificuldade de monitorar vários acontecimentos simultaneamente na sala de aula, transformando o ambiente de ensino em um ambiente caótico (DA SILVA TR, 2016, et al; CHANG ML, 2009; RAVER CC, et al., 2008). Uma parte dos transtornos psiquiátricos são responsáveis pela causa de afastamentos entre os professores, dentre esses transtornos pode destacar a depressão e a ansiedade como as mais comuns, podendo ser intensificadas pela violência na sala de aula e o excesso de trabalho. Há uma parte dos docentes que resistem ao afastamento para tratar a depressão, isso ocorre pelo fato deles não admitirem que estão doentes, por vergonha de admitir que precisam de tratamento médico especializado e por medo de perdas salariais (GASPARINI SM, et al., 2006).

Estudos tem demonstrado a relevância de se pesquisar sobre depressão em professores, mesmo não tendo a atenção necessária dos pesquisadores, uma vez que afeta diretamente o bem-estar dos mesmos, interferindo nos processos de trabalho, incluindo o processo de ensino-aprendizagem, impactando diretamente os estudantes (LIMA MF e LIMA-FILHO DO, 2009). Um estudo realizado na faculdade UFM que examinou a ligação entre a depressão, a violência escolar e a ruptura dos laços pedagógicos, sobressaltou o fato da depressão em professores ser um tema que não tenha tido a atenção necessária dos pesquisadores, e necessita de uma amplificação de estudos sobre esse tema (GASPARINI SM, et al., 2005). Diante do exposto e da relevância da temática proposta o presente estudo tem por objetivo reunir e sistematizar resultados de investigações cientificas relacionados a prevalência de depressão em professores.

\section{MÉTODOS}

Revisão integrativa da literatura sobre depressão em professores. Para o desenvolvimento da pesquisa foram executadas as seguintes etapas: elaboração da questão norteadora, busca na literatura, seleção dos artigos e coleta de dados, análise crítica dos estudos incluídos, discussão dos resultados, e apresentação da revisão integrativa (SOUZA MT, et al., 2010). A pesquisa partiu da seguinte questão norteadora: Qual prevalência de depressão em professores? A coleta de dados ocorreu em setembro de 2019 e foram utilizadas na seleção dos artigos, as seguintes bases de dados eletrônicos: Literatura Latino-Americana em Ciências da Saúde (LILACS) e US National Library of Medicine (PubMed). Empregaram-se os Descritores em Ciências da Saúde (DeCS): Depressão, Professor e Saúde Ocupacional, no idioma inglês (Depression, Teachers, Occupational Health), combinados com o operador booleano (AND), com recorte temporal dos últimos 10 anos, foi possível a captura de 50 artigos. Na Tabela 1 encontra-se o roteiro de busca utilizado para a composição da amostra, que consta a quantidade de artigos utilizados e em qual base de dados foram encontrados.

Tabela 1 - Distribuição dos estudos capturados segundo combinação dos descritores.

\begin{tabular}{lcc}
\hline Descritores Combinados & Estudos encontrados em LILACS & Estudos encontrados em PubMed \\
\hline Depression AND Teachers & 13 & 37 \\
AND Occupational Health & 13 & 37 \\
\hline
\end{tabular}

Fonte: Galinari PC, et al., 2019.

Para seleção dos artigos foram estabelecidos critérios de inclusão e exclusão. Priorizou-se a inclusão de artigos disponíveis na íntegra e que respondessem à pergunta proposta por esta revisão. Como critérios de exclusão artigos não disponíveis na integra, teses e dissertações. O processo de análise e síntese dos artigos foi embasado no instrumento elaborado e validado por Ursi ES e Gavão CM (2006), e realizado de forma descritiva, possibilitando observar, contar, descrever e classificar os dados, com o intuito de reunir o conhecimento produzido sobre o tema explorado na revisão, sendo apreendidas as seguintes informações: autoria dos artigos, ano de publicação, objetivos, temática, resultado e considerações relevantes. Para a avaliação e apresentação dos artigos incluídos na revisão, foi proposta a construção de um quadro comparativo enfocando as principais informações levantadas, as quais foram discutidas posteriormente em categorias temáticas de análise. Os artigos revisados estão apresentados no (Quadro 1), que contém a procedência, título do artigo, autores, períodico (volume, $\mathrm{n}^{\circ}$, página, ano), objetivos e temática, resultados e considerações.

REAEnf/EJNC | Vol.2 | e2546 | DOI: https://doi.org/10.25248/REAenf.e2546.2020 Página 3 de 8 
Quadro 1 - Artigos levantados nas bases de dados LILACS e PubMed.

\begin{tabular}{|c|c|c|c|c|c|}
\hline Procedência & Título do artigo & Autores & $\begin{array}{c}\text { Periódico } \\
\left(\begin{array}{c}\text { (vol, } \mathrm{n}^{\circ}, \text { pág } \\
\text { ano })\end{array}\right.\end{array}$ & Objetivos & Temática/ Resultados / Considerações \\
\hline PubMed & $\begin{array}{l}\text { Occupational } \\
\text { stress, anxiety } \\
\text { and depression } \\
\text { among Egyptian } \\
\text { teachers. }\end{array}$ & $\begin{array}{l}\text { Desouky D, } \\
\text { Allam H. }\end{array}$ & $\begin{array}{l}\text { J Epidemiol } \\
\text { Glob Health. } \\
2017 \text { Sep; } \\
7(3): 191-198\end{array}$ & $\begin{array}{l}\text { Avaliar a prevalência de } \\
\text { depressão e ansiedade entre } \\
\text { professores egípcios. }\end{array}$ & $\begin{array}{l}\text { Foi realizado um estudo transversal com } 568 \text { professores egípcios. Os } \\
\text { entrevistados preencheram a escala de ansiedade de Taylor e o } \\
\text { Inventário de Depressão de Beck (BDI) que foram utilizados para } \\
\text { avaliar, ansiedade e depressão, respectivamente. A prevalência de } \\
\text { ansiedade e depressão entre os professores foi (100\%, } 67,5 \% \text { e } 23,2 \%) \text {, } \\
\text { respectivamente. Os escores de ansiedade e depressão foram } \\
\text { significativamente maiores entre professores com idade acima de } 40 \\
\text { anos, professoras do ensino fundamental, com salário inadequado, } \\
\text { maior experiência de ensino, maior qualificação e maior carga de } \\
\text { trabalho. }\end{array}$ \\
\hline PubMed & $\begin{array}{l}\text { Teachers' } \\
\text { wellbeing and } \\
\text { depressive } \\
\text { symptoms, and } \\
\text { associated risk } \\
\text { factors: A large } \\
\text { cross sectional } \\
\text { study in English } \\
\text { secondary } \\
\text { schools. }\end{array}$ & $\begin{array}{l}\text { Kidger J, et } \\
\text { al. }\end{array}$ & $\begin{array}{l}\text { J Affect } \\
\text { Disord. } 2016 \\
\text { Mar } \\
1 ; 192: 76-82 .\end{array}$ & $\begin{array}{l}\text { Examinar a prevalência de } \\
\text { depressão } \\
\text { autorreferida e fatores de risco } \\
\text { associados em uma grande } \\
\text { amostra de professores do } \\
\text { ensino médio. }\end{array}$ & $\begin{array}{l}\text { Professores }(\mathrm{n}=555) \text { em } 8 \text { escolas preencheram questionários de } \\
\text { autorrelato. Os níveis de bem-estar dos professores (Escala de Bem- } \\
\text { Estar Mental Warwick Edinburgh-WEMWBS) e sintomas depressivos } \\
\text { (Questionário de Saúde do Paciente-PHQ-9) foram medidos e as } \\
\text { associações entre essas medidas e fatores relacionados à escola foram } \\
\text { examinadas usando modelos de regressão multivariável em vários } \\
\text { níveis. Os resultados mostraram que bem-estar é baixo e } \\
\text { os sintomas depressivos são altos entre os professores. Intervenções } \\
\text { destinadas a melhorar sua saúde mental podem se concentrar na } \\
\text { redução do estresse relacionado ao trabalho e no aumento do apoio } \\
\text { disponível. }\end{array}$ \\
\hline
\end{tabular}

REAEnf/EJNC | Vol.2 | e2546 | DOI: https://doi.org/10.25248/REAenf.e2546.2020 Página 4 de 8 


\begin{tabular}{|c|c|c|c|c|c|}
\hline LILACS & $\begin{array}{l}\text { Mental disorders } \\
\text { in university } \\
\text { teachers: study } \\
\text { in a service of } \\
\text { medical } \\
\text { investigation. }\end{array}$ & $\begin{array}{l}\text { Batista JB } \\
\text { VI, et al. }\end{array}$ & $\begin{array}{l}\text { Rev. pesqui. } \\
\text { cuid. fundam. } \\
\text { (Online); } \\
\text { 8(2): 4538- } \\
\text { 4548, abr.- } \\
\text { jul.2016. }\end{array}$ & $\begin{array}{l}\text { Identificar os transtornos } \\
\text { mentais que mais provocam } \\
\text { afastamento de docentes em } \\
\text { uma instituição de ensino } \\
\text { superior. }\end{array}$ & $\begin{array}{l}\text { Estudo retrospectivo, de natureza documental, desenvolvido em } \\
\text { instituição de ensino superior do município de João Pessoa-PB. } \\
\text { Amostra constituída por } 254 \text { fichas de docentes atendidos em um } \\
\text { serviço de perícia médica da instituição selecionada. Os dados obtidos } \\
\text { foram analisados quantitativamente por frequência e percentual. Quase } \\
\text { toda a amostra foi composta por docentes do sexo feminino, com faixa } \\
\text { etária entre } 40 \text { e } 49 \text { anos. A depressão foi responsável por } 53 \% \text { dos } \\
\text { afastamentos de professores, a esquizofrenia (12\%), o transtorno } \\
\text { bipolar (10\%), a reação aguda ao estresse ( } 8 \%) \text {, a ansiedade (7\%), os } \\
\text { transtornos delirantes (4\%) e outros (8\%). O transtorno mental que mais } \\
\text { acomete a categoria docente pesquisada é a depressão. Portanto, são } \\
\text { necessárias novas investigações que possam analisar a gravidade } \\
\text { dessa problemática. }\end{array}$ \\
\hline LILACS & $\begin{array}{l}\text { Evaluation of } \\
\text { stress and } \\
\text { depression } \\
\text { levels in } \\
\text { teachers of } \\
\text { public schools in } \\
\text { the city of } \\
\text { Francisco } \\
\text { Beltrão - PR }\end{array}$ & $\begin{array}{l}\text { Scandolara } \\
\text { TB, et al. }\end{array}$ & $\begin{array}{l}\text { Arq. ciências } \\
\text { saúde } \\
\text { UNIPAR; } \\
\text { 19(1): } 31-38, \\
\text { jan-abr. } \\
2015 .\end{array}$ & $\begin{array}{l}\text { Detectar a percentagem de } \\
\text { professores da rede pública } \\
\text { que apresentam altos níveis de } \\
\text { estresse e depressão. }\end{array}$ & $\begin{array}{l}\text { Foi realizado um estudo prospectivo, experimental e longitudinal, com o } \\
\text { objetivo de detectar a percentagem de professores da rede pública que } \\
\text { apresentam altos níveis de estresse e depressão e alertá-los sobre os } \\
\text { problemas decorrentes destas patologias. Foram utilizados dois } \\
\text { questionários validados de autopreenchimento para verificar a } \\
\text { severidade de distúrbios psiquiátricos não psicóticos, relacionados ao } \\
\text { estresse e depressão. Os escores foram obtidos a partir da somatória } \\
\text { das questões respondidas pelos professores no questionário GHQ-12 e } \\
\text { no Inventário de Beck. Dos } 106 \text { professores participantes da pesquisa, } \\
46,2 \% \text { deles sofrem de algum nível de estresse psicossomático e } 21,7 \% \\
\text { possuem características de depressão. }\end{array}$ \\
\hline LILACS & $\begin{array}{l}\text { Depression as } \\
\text { cause of work } \\
\text { leave: a study } \\
\text { with elementary } \\
\text { school teachers }\end{array}$ & $\begin{array}{l}\text { Batista JB } \\
\text { VI, et al. }\end{array}$ & $\begin{array}{l}\text { Psico (Porto } \\
\text { Alegre); } \\
44(2): 257- \\
\text { 262, abr.-jun. } \\
2013 .\end{array}$ & 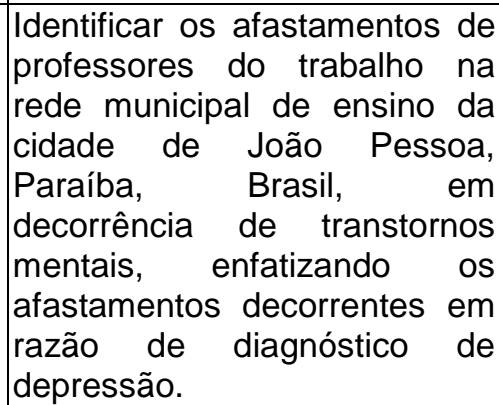 & $\begin{array}{l}\text { Chamando a atenção para a prevalência da depressão na categoria } \\
\text { docente, este estudo identificou afastamentos do trabalho de } \\
\text { professores da rede municipal da cidade de João Pessoa, Paraíba, } \\
\text { Brasil, em decorrência de transtornos mentais, especificamente, } \\
\text { depressão. É um estudo descritivo, retrospectivo, transversal, } \\
\text { quantitativo, baseado no universo de } 414 \text { fichas médicas individuais de } \\
\text { professores, entre os anos de } 1999 \text { e } 2006 \text {, cujos diagnósticos para o } \\
\text { afastamento do trabalho correspondiam a transtornos mentais. Os } \\
\text { resultados mostram que, das } 414 \text { licenças no período estudado, } 211 \\
(51 \%) \text { foram devido à depressão. }\end{array}$ \\
\hline
\end{tabular}

REAEnf/EJNC | Vol.2 | e2546 | DOI: https://doi.org/10.25248/REAenf.e2546.2020 Página 5 de 8 


\section{RESULTADOS e DISCUSSÃO}

A amostra final foi constituída por 5 (cinco) artigos, sendo 2 artigos da PubMed e 3 da LILACS, o que demonstra incipiência de estudos relacionados a essa temática, com média inferior a um estudo por ano, considerando o período de publicação dos artigos incluídos. As temáticas centrais foram prevalência da depressão entre professores e afastamento do trabalho relacionado à depressão. Quanto ao método das pesquisas, todos se configuram por delineamento transversal.

No que tange à autoria dos estudos, foi identificado à participação de médicos e enfermeiros professores universitários nas áreas de Saúde Pública e Medicina de Família e Comunidade, e outros profissionais de saúde vinculados a programas de pós-graduação stricto sensu.

Os achados desse estudo demonstram alta prevalência de depressão e sintomas depressivos nos professores. Dois dos estudos analisados utilizaram a Escala de Beck, a fim de avaliar a prevalência de sintomas depressivos nessa população (DESOUKY D e ALLAM H, 2017; SCANDOLARA TB, et al., 2015).

Originalmente desenvolvido por Beck, Erbaugh, Ward, Mendelson e Mock em 1961, o Inventário de Depressão de Beck (Beck Depression Inventory) - BDI trata-se de uma escala de auto-relato, que mede a intensidade da depressão, assim como dos sintomas depressivos. Ela é composta por 21 itens, quantificados e somados. Quanto maior o valor total maior o grau de depressão (BECK AT e STEER RA, 1987).

No Egito, estudo realizado com professores de escola primária e secundária, no período de janeiro a março de 2017 apontou que a prevalência de sintomas depressivos leves, moderados e severos entre os professores estudados foi de 19,7\%, 2,8\% e 0,7\% respectivamente (DESOUKY D e ALLAM H, 2017). No município de Francisco Beltrão, no estado do Paraná, foi realizado um estudo prospectivo com 106 professores da rede pública demonstrando resultados semelhantes, com prevalência de sintomas depressivos leves em 13,2\%, moderados em 7,5\% e severos 0,9\% (SCANDOLARA TB, et al., 2015).

De acordo com o Manual diagnóstico e estatístico de transtornos mentais (DSM-V), o diagnóstico da depressão segundo a observação clínica é feito a partir da presença de diversos sintomas, sendo perda ou ganho de peso ou de apetite, fadiga, insônia ou hipersônia, diminuição da concentração, pensamento de morte ou suicídio, humor deprimido, retardo ou agitação psicomotora, anedonia e sentimento de culpa (AMERICAN PSYCHIATRIC ASSOCIATION, 2014).

Os sintomas depressivos, em todos os estudos analisados, estão associados ao sexo feminino, as taxas de sintomas leves, moderados e graves em mulheres foram relativamente maiores do que as taxas apresentadas em professores do sexo masculino (DESOUKY D e ALLAM H, 2017; SCANDOLARA TB, et al., 2015; KIDGER J, et al., 2016). Também foi observada, maior prevalência de afastamentos do trabalho, por sintomas relacionados à depressão entre as mulheres (BATISTA JBV, et al., 2016; BATISTA JBV, et al., 2013).

Muitos estudos epidemiológicos avaliaram a depressão nas mulheres aproximadamente duas vezes mais prevalente que em homens (ANGST J, et al., 2002; WEISSMAN MM, et al., 1996). Maior prevalência de depressão em mulheres pode estar relacionada ao fato da maior percepção das mulheres para com a própria saúde, assim como maior conhecimento dessas acerca de sintomas físicos e, em consequência, maior procura dos serviços de saúde (PINHEIRO RS, et al., 2002). Essa maior prevalência das mulheres, ocorre também em outras formas de manifestações depressivas, é o caso da distimia e da depressão menor, mesmo que ocorram de maneira menos acentuada (ANGST J, et al., 2002).

Quanto à faixa etária, a análise dos estudos demonstra maior prevalência de depressão e sintomas depressivos nas idades acima de 40 anos (BATISTA JBV, et al., 2013; SCANDOLARA TB, et al., 2015; BATISTA JBV, et al., 2016; DESOUKY D e ALLAM H, 2017). Maior prevalência de afastamento do trabalho relacionado à depressão, também foi observado nessa faixa etária (BATISTA JBV, et al., 2016; BATISTA JBV, et al., 2013). Como justificativa desse fenômeno, estudos tem relacionado o tempo de carreira na profissão de professor como desencadeante de sintomas depressivos mais aparentes nessas idades, devido a um maior tempo de exposição ao enfrentamento de problemas relacionados à ocupação, como o

REAEnf/EJNC | Vol.2 | e2546 | DOI: https://doi.org/10.25248/REAenf.e2546.2020 Página 6 de 8 
ambiente de trabalho, condições organizacionais, estabilidade no emprego, salário, relações sociais no trabalho, carga de trabalho, autonomia, reconhecimento e valorização profissional (GASPARINI SM, et al., 2006; BATISTA JBV, et al., 2016)

Os distúrbios depressivos estiveram relacionados a mais da metade dos afastamentos do trabalho, por transtornos mentais, entre os professores, com tendência crescente na quantidade de afastamentos anuais, decorrentes de transtornos mentais (BATISTA JBV, et al., 2016; BATISTA JBV, et al., 2013).

Tem sido demonstrado que o ensino é uma ocupação altamente estressante se destacando como fatores desencadeantes de estresse a carga horária excessiva ou múltipla jornada de trabalho, elevado número de alunos em sala, causando exaustão emocional e diminuição do sentimento de realização profissional, prejudicando a saúde mental e física do educador e dos alunos, podendo acarretar até mesmo em abandono da profissão por parte do professor (LEVY GCTM, et al., 2009).

A carga de afastamento do trabalho por sintomas depressivos e depressão foi associada à redução da produtividade e do desempenho do trabalhador, com forte impacto em sua vida e limitação de sua contribuição à sociedade (BATISTA JBV, et al., 2013). O afastamento dos docentes acontece por muitos períodos, pelos mesmos motivos e repetidas vezes. A volta ao trabalho ocasiona a piora da sintomatologia, podendo supor que o retorno ao ambiente escolar, ocorre nas mesmas circunstâncias equivalentes aquelas que originaram os afastamentos, a falta de políticas adequadas para o acontecimento de retorno é um agravante. (GLINA DMR, et al., 2001).

Há poucos estudos em relação a afastamentos por transtornos mentais, mas há uma concordância que a preparação para o regresso ao trabalho na escola seria uma oportunidade de participação dos inúmeros atores, que poderia ser no caso a equipe gestora da instituição escolar, responsável pelo regresso ao ambiente escolar, que poderia atuar como mediador entre o professor e o departamento de licenças medica, e o sujeito central no processo o próprio professor (GLINA DMR, et al., 2001).

As manifestações clínicas da depressão comprometem nas relações pessoais e familiares do indivíduo, gerando impacto determinante no desempenho do trabalho, estando associada a diminuição do desempenho e da produtividade com repercussões sociais negativas, impactando diretamente a vida do indivíduo que a desenvolve (JARDIM S, 2011).

A depressão tanto pode ser compreendida como uma doença, com características e diagnóstico próprios, como também pode estar associada a outros transtornos emocionais, de etiologia, sintomatologia e tratamento mais complexos, como a Síndrome de Burnout. Essa síndrome está diretamente relacionada às atividades laborais e ao estresse no ambiente de trabalho, e afeta trabalhadores de diversas áreas, especialmente os que lidam diretamente com atendimento ao público, também gerando alta carga de absenteísmo. Em vista disso, o afastamento do profissional por decorrência do trabalho deve ser investigado, com o fim de descobrir o fator desencadeador, proporcionar tratamento adequado e evitar 0 acréscimo de novos professores deprimidos. Ter um professor em estado deprimido no ambiente de aula pode prejudicar a relação professor e aluno, além de danos institucionais (BATISTA JBV, et al., 2013).

\section{CONCLUSÃO}

A presente revisão integrativa possibilitou caracterizar a produção científica acerca da prevalência de depressão em professores. Apesar de ter sido incluído somente cincos artigos, pode se perceber maior prevalência de depressão e sintomas depressivos em professores nas idades acima de 40 anos, no sexo feminino. Também se destaca como a principal causa de afastamento do trabalho relacionado a transtornos mentais. Consideramos de fundamental importância a criação de medidas preventivas frente a depressão, visto o crescente aumento do transtorno depressivo em professores, seu desenvolvimento e possíveis desfechos. A criação de tais medidas se faz necessária, uma vez que os professores atuam diretamente na qualidade de ensino e a debilitação deste profissional pode resultar em queda da mesma, sendo de grande dano ao país. Ressalta-se ainda a necessidade da ampliação de estudos na área, com aplicação de questionários e acompanhamento dos investigados, a fim de se quantificar e qualificar melhor os dados referentes ao processo do transtorno depressivo nos docentes em ambientes de trabalho, contribuindo para a elaboração de medidas preventivas mais adequadas e eficazes. 


\section{REFERÊNCIAS}

1. AMERICAN PSYCHIATRIC ASSOCIATION. Manual diagnóstico e estatístico de transtornos mentais - DSM-5. 5. ed. Porto Alegre: Artmed, 2014.

2. ANGST J, et al. Gender differences in depression. European Archives of Psychiatry and Clinical Neuroscience, 2002; 252(5): 201-209.

3. ASSOCIAÇÂO AMERICANA DE PSIQUIATRIA. Manual diagnóstico e estatístico de transtornos mentais- DSM. 5 ed. Porto Alegre: Artmed, 2014: 992p.

4. ATKINSON LR, et al. Introdução à Psicologia de Hilgard. Tradução Bueno, D.; 13. Ed. Porto Alegre: Artmed, 2002. p. 562-563.

5. BATISTA JBV, et al. Depression as cause of work leave: a study with elementary school teachers. Psico, 2013; 44(2): 257-262.

6. BATISTA JBV, et al. Mental disorders in university teachers: study in a service of medical investigation. Revista de Pesquisa: Cuidado é Fundamental Online, 2016; 8(2): 4538-48.

7. BECK AT, STEER RA. Beck Depression Inventory Manual. Toronto, Canada: Psychological Corp, Harcourt, Brace, Jovanovich; 1987; 25p.

8. BOURBONNAIS R, et al. Job strain, psychological distress, and burnout in nurses. American Journal of Industrial Medicine, 1998; 34(1): 20-28.

9. CHANG ML. Uma perspectiva de avaliação do desgaste dos professores: Examinando o trabalho emocional dos professores. Revisão de Psicologia Educacional. 2009; 21(3): 193-218.

10. DA SILVA TR, DE CARVALHO EA. Depressão Em Professores Universitários: Uma Revisão Da Literatura Brasileira. Revista Uningá Review, 2016; 28(1): 113-117.

11. DESOUKY D, ALLAM H. Occupational stress, anxiety and depression among Egyptian teachers. Journal of Epidemiology and Global Health, 2017; 7(3): 191-198.

12. GASPARINI SM, et al. O professor, as condições de trabalho e os efeitos sobre sua saúde. Educação e Pesquisa, São Paulo, 2005; 31(2): 189-199.

13. GASPARINI SM, et al. Prevalência de transtornos mentais comuns em professores da rede municipal de Belo Horizonte, Minas Gerais, Brasil. Cad. Saúde Pública, Rio de Janeiro, 2006; 22(12): 2679-2691.

14. GATTI BA, et al. Políticas docentes no Brasil: um estado da arte. Brasília: UNESCO, 2011: 300 p.

15. GLINA DMR, et al. Saúde mental e trabalho: uma reflexão sobre o nexo com o trabalho e o diagnóstico com base na prática. Cadernos de Saúde Pública, Rio de Janeiro, 2001; 17(3): 607- 616.

16. JARDIM, S. (2011) Depressão e trabalho: ruptura de laço social. Rev. bras. saúde ocup, 2011; 36(123): 84-92.

17. JOHNSRUD LK, HECK RH. Faculty worklife: establishing benchmarks across groups. Research in Higher Education, 1998; 39: 539.

18. KIDGER J, et al. Teachers' wellbeing and depressive symptoms, and associated risk factors: A large cross sectional study in English secondary schools. Journal of Affective Disorders, 2016; 192: 76-82.

19. LASSER J, KAHN J. Depresion.OrganizationInternacionale de Trabajo, Enciclopedia de salud y seguridadeneltrabajo. 1998, 34.86.

20. LEVY GCTM, et al. Síndrome de Burnout em professores da rede pública. Prod., São Paulo, 2009; 19 (3): 458-465.

21. LIMA MF, LIMA-FILHO DO. Condições de trabalho e saúde do/a professor/a universitário/a. Ciências \& Cognição, 2009; 14(3): 74-89.

22. NASCIMENTO DCD, et al. Depressão em idosos residentes em uma instituição asilar na cidade de Juazeiro do Norte, Ceará, Brasil. J. Manag. Prim. Health Care, 2013; 4(3): 146 - 150.

23. NOGUEIRA DXP. Educação: carinho e trabalho. Burnout, a síndrome da desistência do educador, que pode levar à falência da educação. Revista Retratos da Escola, Brasília, 2012; (6)11: 507-509.

24. ORGANIZAÇÃO MUNDIAL DE SAÚDE [homepage internet]. Depressão. 2019.

25. PINHEIRO RS, et al. Gênero, morbidade, acesso e utilização de serviços de saúde no Brasil. Ciênc Saúde Coletiva 2002; 7(4): 687-707.

26. RAVER CC, et al. Melhorando os processos em sala de aula pré-escolar: resultados preliminares de um estudo randomizado implementado nas configurações do Head Start. Pesquisa sobre a primeira infância trimestral, 2008; 23(1): 10-26.

27. SCANDOLARA TB, et al. Avaliação Dos Niveis De Estresse EDepressão Em Professores Da Rede PublicaDo Municipio De Francisco Beltrão - PR. Arq. Cienc. Saúde UNIPAR, 2015; 19(1): 31-38.

28. SOUZA MT, et al. Revisão Integrativa: o que é e como fazer. Einstein, São Paulo, 2010; 8(1): 102-106.

29. URSI ES, GAVÃO CM. Prevenção de lesões de pele no perioperatório: revisão integrativa da literatura. Rev LatinoAm Enfermagem. 2006; 14(1):124-31.

30. WEISSMAN MM, et al. Cross-National Epidemiology of Major Depression and Bipolar Disorder. JAMA: The Journal of the American Medical Association, 1996; 276(4): 293. 